Case Report

\title{
Thyroid Eye Disease due to Immune Reconstitution Inflammatory Syndrome as a Consequence of Antiretroviral Therapy in the Setting of AIDS
}

\author{
Ravali Nallu, ${ }^{1}$ Parvathy Madhavan ${ }^{D},{ }^{1}$ Lisa Chirch, ${ }^{2}$ and Pooja Luthra ${ }^{1}$ \\ ${ }^{1}$ Department of Medicine, Division of Endocrinology and Metabolism, University of Connecticut, School of Medicine, \\ Farmington, CT, USA \\ ${ }^{2}$ Department of Medicine, Division of Infectious Diseases, University of Connecticut, School of Medicine, Farmington, CT, USA
}

Correspondence should be addressed to Parvathy Madhavan; madhavan.parvathy@gmail.com

Received 19 August 2019; Revised 2 January 2020; Accepted 24 January 2020; Published 12 February 2020

Academic Editor: Mihail A. Boyanov

Copyright (c) 2020 Ravali Nallu et al. This is an open access article distributed under the Creative Commons Attribution License, which permits unrestricted use, distribution, and reproduction in any medium, provided the original work is properly cited.

We describe a case of worsening Graves' orbitopathy due to immune reconstitution inflammatory syndrome (IRIS) in a 38-yearold HIV-infected male after beginning ART (antiretroviral therapy). Two years after initiation of ART, the patient developed symptoms of hyperthyroidism and thyroid eye disease (TED) or Graves' orbitopathy (GO). Thyroid iodine uptake scan was consistent with Graves' disease. The CT scan of the orbits revealed minimal right-sided proptosis, consistent with GO. He was treated with methimazole and a short course of high-dose prednisone for GO. Thyroid function tests normalized, and eye symptoms eventually stabilized. This case demonstrates the importance of awareness and early recognition of IRIS in its many forms, as it has significant therapeutic implications.

\section{Background}

Antiretroviral therapy (ART) has dramatically improved the morbidity and mortality of patients infected with the human immunodeficiency virus (HIV), but it may also increase the risk of immune reconstitution inflammatory syndrome (IRIS). IRIS is characterized by a pathological inflammatory response to a pre-existing pathogen or antigen and paradoxical worsening of clinical status, after initiation of ART [1]. IRIS develops in $10-25 \%$ of patients with AIDS receiving ART [1]. Graves' disease (GD) occurs in 1-2\% of patients with HIV as a late manifestation of IRIS [2]. GD is an autoimmune disease caused by production of IgG autoantibodies against the thyrotropin (TSH) receptor, binding and activating it and causing autonomous production of thyroid hormone [3]. Thyroid eye disease (TED) or Graves' orbitopathy (GO) occurs in about $25 \%$ of all patients with GD [1]. The incidence and prevalence of TED as a manifestation of IRIS is unknown. We discuss the rare occurrence of TED due to GD, as a result of IRIS, in a patient with AIDS.

\section{Case}

A 38-year-old male diagnosed with HIV-1 infection in 2014 was treated with ART. He did not have a prior history of thyroid disease although his sister had a history of hyperthyroidism. Physical examination was unremarkable. His baseline CD4+ T-cell count was very low at 32 cells/ $\mu$ l with a plasma HIV RNA of 213,000 copies/ml. The patient was started on ART with emtricitabine/rilpivirine/tenofovir disoproxil fumarate at an outside facility. On evaluation at our institution for a second opinion, he was transitioned to emtricitabine/tenofovir disoproxil fumarate, darunavir, ritonavir, and dolutegravir based on resistance testing that revealed reverse transcriptase mutations at the M184 and Y181 sites. This regimen resulted in complete suppression of HIV RNA within the first 12 weeks of therapy, accompanied by a significant rise of $\mathrm{CD} 4+\mathrm{T}$-cell count.

At presentation, the TSH level was normal at $1.34 \mathrm{mIU} / \mathrm{l}$ $(0.27-4.20 \mathrm{mIU} / \mathrm{l})$. Thyroid antibody levels were not available at that time. 
Two years after initiation of ART (2016), the patient developed insomnia, palpitations, heat intolerance, unintentional $20 \mathrm{lbs}$ weight loss, and bulging of the eyes. On exam, significant bilateral exophthalmos was noted, along with a prominent thyroid gland.

See Table 1 for laboratory data.

The thyroid iodine I-131 uptake scan showed diffuse increase in the uptake without evidence of hot or cold nodules.

The patient was diagnosed with GD with significant TED and started on antithyroid therapy with methimazole and a beta-blocker. The patient stopped the antithyroid medication after a few months and noticed further progression of eye symptoms. A CT scan of the orbits revealed minimal right-sided proptosis consistent with TED (Supplementary Materials available here). He was restarted on the methimazole and a short course of high-dose prednisone for TED. Thyroid function tests normalized, and eye symptoms eventually stabilized.

\section{Discussion}

HIV affects the immune system by specifically infecting the CD4+ T-cells, resulting in the development of a wide variety of opportunistic infections. Since the development and widespread use of highly active ART in the 1990s, there has been a significant reduction in the morbidity and mortality associated with the disease. However, a small subset of patients treated with ART, in particular those with very low nadir CD4+ T-cell counts, experience a paradoxical clinical deterioration associated with the recovery of the immune system. This has been described as an entity known as IRIS and is believed to affect about $10-40 \%$ of patients beginning ART [4]. This presentation is believed to be related to the recovery of immune function and restoration of the ability to mount an inflammatory response to both infectious and noninfectious agents. The exact mechanism is not completely understood. Resurgence of autoimmune conditions has most commonly been shown to occur in the later stages of AIDS [5].

GD as a consequence of IRIS after initiation of ART is well described [6]. It is thought to behave similarly to "conventional" GD and therefore should be treated in a similar manner. However, extrathyroidal manifestations of GD, such as TED, are not well characterized. TED is an inflammatory condition affecting the orbital tissue and occurs in about $25 \%$ of patients with GD [1]. It is characterized by lymphocytic infiltration of the extraocular muscles and orbital adipose tissue, orbital fibroblast proliferation and differentiation, deposition of hyaluronic acid and glycosaminoglycans, and de novo adipogenesis [7]. The pathogenesis of this condition is believed to be secondary to a complex interplay between genetic and lifestyle factors (e.g., cigarette smoking) leading to an inflammatory cascade, with involvement of autoantibodies such as TSH receptor (TSHR) and insulin-like growth factor-1 (IGF-1) receptor [8].

TED has a spectrum of clinical manifestations and can range from mild disease to severe extraocular muscle dysfunction and vision loss due to exposure keratopathy or
TABLE 1: Laboratory data.

\begin{tabular}{lccrc}
\hline & 2014 (Pre-ART) & 2016 (Post-ART) & 2017 & 2018 \\
\hline TSH & 1.34 & $<0.01$ & 1.06 & 0.54 \\
Free T4 & N/A & 2.38 & 0.99 & N/A \\
TT3 & N/A & 526 & 139 & 128 \\
TSI & N/A & $>500 \%$ & N/A & N/A \\
CD4 & 32 & 396 & 343 & 344 \\
\hline
\end{tabular}

TSH: $\quad 0.35-4.94 \mathrm{mIU} / \mathrm{ml}$. Free T4:0.61-1.82 ng/dl. Total T3 (TT3): $48-178 \mathrm{ng} / \mathrm{dl}$. Thyroid-stimulating immunoglobulins (TSI) $<122 \%$. CD4+ T-cells, 430-1800 cells/ $\mu$; N/A, not available.

optic nerve compression [8]. ART treatment increases the CD4+ T-cell count in 2 phases: initial increase in the memory CD4+ T-cells that occurs in the first few months of therapy; and a slower and steady increase over many months to years, in the naive CD4+ T-cells, which originate from the thymus [9]. The CD4+ T-cells mature in the thymus, and cells which are capable of recognizing the major histocompatibility complex (MHC) molecules associated with foreign antigens enter the periphery, whereas those reacting with self-antigens undergo apoptosis [10]. However, initiation of ART causes intense regeneration of the thymus, resulting in an insufficient suppression of the autoreactive cells. This leads to the manifestation of a wide variety of autoimmune phenomenon including GD. Even though the functionality of the thymus is thought to decrease with age, recent reports have shown that it may remain active longer in HIV-infected individuals [11].

Another hypothesis for expansion of autoreactive $\mathrm{T}$ lymphocytes and their redistribution in IRIS is due to "molecular mimicry" due to homology between TSH-R and immunogenic HIV-related proteins [8]. Other predisposing factors associated with the development of IRIS include advanced immunodeficiency at baseline, more pronounced increase in CD4+ T lymphocytes, and genetic susceptibility [5].

In 2000, Jubault et al. [12] published a series of five patients, median age of 41 years, who developed GD after the initiation of ART. None of the patients had prior autoimmune conditions. In 2009, Vos et al. [13] described 3 similar cases and performed a literature review that revealed 13 reported cases of GD secondary to IRIS. Wong et al. [14] demonstrated a similar course in $13 \mathrm{HIV}$-infected patients of Chinese origin. In all of these studies, there was a direct relationship between the rise of the CD4+ T-cell count and corresponding suppression of the viral load, after beginning therapy with ART, and occurrence of GD. This was clearly demonstrated in our case as well. Chen et al. [15] published a cohort of $17 \mathrm{HIV}$ affected patients, predominantly African American, who developed autoimmune thyroid disease (AITD) as a late complication of IRIS, after treatment with ART.

Management of TED can be challenging in the presence of HIV infection. Patients with poorly controlled thyroid function have more severe manifestations of TED [16]. In addition, certain medications increase the possibility of thyroid dysfunction. In a large single-center cohort of HIVinfected patients, the prevalence of clinically apparent hyperthyroidism was $1.01 \%$, most commonly associated with use of non-NRTI (NNRTI), particularly efavirenz [17]. On 
the other hand, there was a high prevalence of subclinical hypothyroidism, with the use of nucleoside reverse transcriptase inhibitor (NRTI), stavudine [18]. It is uncertain if these medications increase the severity of TED, but these associations should be kept in mind when choosing ART regimens. It is also important to consider interactions between ART and medications used in patients with TED. NNRTIs may induce cytochrome P450 (e.g., efavirenz), whereas ritonavir-boosted protease inhibitors (PI) inhibit cytochrome P450. Ritonavir and cobicistat (a pharmacologic booster) can significantly increase the concentration and effect of glucocorticoids, potentially leading to Cushing's syndrome [19]. It is important to closely monitor patients with HIV infection on high-risk medications for any manifestations of IRIS for prompt diagnosis and management.

\section{Conclusion}

Our case demonstrates the importance of being vigilant about the occurrence of various autoimmune conditions as manifestations of IRIS in patients receiving ART. These may occur even 1-3 years after initiation of therapy, as described in our case [10]. The management of GD and TED in the presence of HIV infection and ART appears to be equivalent to standard treatment with particular attention to potential drug interactions. Timely recognition and treatment may help to reduce the complications associated with these disorders.

\section{Disclosure}

This case report was presented in the 2018 Endocrine Society Meeting in Chicago.

\section{Conflicts of Interest}

The authors declare that they have no conflicts of interest.

\section{Supplementary Materials}

Image of the CT scan of the orbit. (Supplementary Materials)

\section{References}

[1] L. J. Haddow, P. J. Easterbrook, A. Mosam et al., "Defining immune reconstitution inflammatory syndrome: evaluation of expert opinion versus 2 case definitions in a South African cohort," Clinical Infectious Diseases, vol. 49, no. 9, pp. 1424-1432, 2009.

[2] K. Mayer, C. J. Hoffman, and T. T. Brown, "Thyroid function abnormalities in HIV infected patients," Clinical Infectious Diseases, vol. 45, no. 4, pp. 488-494, 2007.

[3] B. McIver and J. C. Morris, "The pathogenesis of Graves' disease," Endocrinology and Metabolism Clinics of North America, vol. 27, no. 1, pp. 73-89, 1998.

[4] M. A. French, P. Price, and S. F. Stone, "Immune restoration disease after antiretroviral therapy," AIDS, vol. 18, no. 12, pp. 1615-1627, 2004.
[5] L. E. Vega, "Espinoza LR HIV infection and its effects on the development of autoimmune disorders," Pharmacological Research, vol. 129, pp. 1-9, 2018.

[6] A. Weetman, "Immune reconstitution syndrome and the thyroid," Best Practice \& Research Clinical Endocrinology \& Metabolism, vol. 23, no. 6, pp. 693-702, 2009.

[7] R. Bahn, "Graves' ophthalmopathy," New England Journal of Medicine, vol. 362, no. 8, pp. 726-738, 2010.

[8] M. R. Edmunds, F. Mellington, R. L. Ford, B. Torlinska, K. Manavi, and K. Boelaert, "Clinical challenges of thyroid eye disease in HIV-positive patients on highly active antiretroviral therapy," The Journal of Clinical Endocrinology \& Metabolism, vol. 100, no. 3, pp. 779-787, 2015.

[9] A. A. Parsa and A. Bhangoo, "HIV and thyroid dysfunction," Reviews in Endocrine and Metabolic Disorders, vol. 14, no. 2, pp. 127-131, 2013.

[10] B. Knysz, M. Bolanowski, M. Klimczak, K. Gladysz, and K. Zwolinska, "Graves' disease as an immune reconstitution syndrome in an HIV-1-positive patient commencing effective antiretroviral therapy: case report and literature review," Viral Immunology, vol. 19, no. 1, pp. 102-107, 2006.

[11] J. M. McCune, R. Loftus, D. K. Schmidt et al., "High prevalence of thymic tissue in adults with human immunodeficiency virus-1 infection," Journal of Clinical Investigation, vol. 101, no. 11, pp. 2301-2308, 1998.

[12] V. Jubault, A. Penfornis, F. Schillo et al., "Sequential occurrence of thyroid autoantibodies and Graves' disease after immune restoration in severely immunocompromised human immunodeficiency virus-1-infected patients," Journal of Clinical Endocrinology \& Metabolism, vol. 85, no. 11, pp. 4254-4257, 2000.

[13] F. Vos, G. Pieters, M. Keuter, and A. van der Ven, "Graves' disease during immune reconstitution in HIV-infected patients treated with HAART," Scandinavian Journal of Infectious Diseases, vol. 38, no. 2, pp. 124-126, 2006.

[14] K. H. Wong, S. S. Chow, W. Lee, and S. S. Lee, "Clinical hyperthyroidism in Chinese patients with stable HIV disease," Clinical Infectious Diseases, vol. 39, no. 8, pp. 1257-1259, 2004.

[15] F. Chen, S. L. Day, R. A. Metcalfe et al., "Characteristics of autoimmune thyroid disease occurring as a late complication of immune reconstitution in patients with advanced human immunodeficiency virus (HIV) disease," Medicine, vol. 84, no. 2, pp. 98-106, 2005.

[16] M. F. Prummel, W. M. Wiersinga, M. P. Mourits, L. Koornneef, A. Berghout, and R. Van der Gaag, "Effect of abnormal thyroid function on the severity of Graves' ophthalmopathy," Archives of Internal Medicine, vol. 150, no. 5, pp. 1098-1101, 1990.

[17] S. Rasul, R. Delapenaha, F. Farhat, J. Gajjala, and S. M. Zahra, "Graves' disease as a manifestation of immune reconstitution in HIV-infected individuals after initiation of highly active antiretroviral therapy," AIDS Research and Treatment, vol. 2011, Article ID 743597, 4 pages, 2011.

[18] G. Madeddu, A. Spanu, F. Chessa et al., "Thyroid function in human immunodeficiency virus patients treated with highly active antiretroviral therapy (HAART): a longitudinal study," Clinical Endocrinology, vol. 64, no. 4, pp. 375-383, 2006.

[19] M. Foisy, E. Yakiwchuk, I. Chiu, and A. Singh, "Adrenal suppression and Cushing's syndrome secondary to an interaction between ritonavir and fluticasone: a review of the literature," HIV Medicine, vol. 9, no. 6, pp. 389-396, 2008. 